Research Article

\title{
Weighted Voronoi Diagram-Based Simulation and Comparative Analysis of Ecosystem Service Coverage: Case Study of the Zhongyuan Urban Agglomeration
}

\author{
Pengyan Zhang, ${ }^{1}$ Wenlong Jing $\mathbb{D}^{2,3,4}$ and Yunzeng Chen $\mathbb{D}^{1}$ \\ ${ }^{1}$ College of Environment and Planning, Henan University, Kaifeng 475004, China \\ ${ }^{2}$ Guangzhou Institute of Geography, Guangzhou 510070, China \\ ${ }^{3}$ Key Laboratory of Guangdong for Utilization of Remote Sensing and Geographical Information System, Guangzhou 510070, China \\ ${ }^{4}$ Guangdong Open Laboratory of Geospatial Information Technology and Application, Guangzhou 510070, China
}

Correspondence should be addressed to Wenlong Jing; jingwl@lreis.ac.cn and Yunzeng Chen; yzchen@henu.edu.cn

Received 27 April 2018; Accepted 5 July 2018; Published 5 August 2018

Academic Editor: Yichun Xie

Copyright @ 2018 Pengyan Zhang et al. This is an open access article distributed under the Creative Commons Attribution License, which permits unrestricted use, distribution, and reproduction in any medium, provided the original work is properly cited.

The future of ecology lies on the promotion of eco-economy, a resource saving and environment-friendly society, and the research of ecosystem services. This article applied weighted Voronoi diagram to simulate the ecosystem service capacity in Zhongyuan Urban Agglomeration, China, using remote sensing and geographic information systems. The results show that (1) compared with the traditional Voronoi diagram, the weighted Voronoi diagram expresses more accurate on the ecosystem service range, (2) the total value of the ecosystem service in the study area was 575.7 billion yuan in 2015. The maximum value was about 253.8 billion yuan greater than the minimum value. The unit forest ecosystem has been improved after the coefficient was modified, and the value of ecosystem services reached $32,638.87$ yuan $/ \mathrm{hm}^{2}$, (3) the service value of construction ecosystem has a great effect on the total value of ecosystem services; the overall pulldown effect of Kaifeng City is most considerable, and (4) the region with strong ecosystem service capacity has strong effect in annexing the nearby areas with weak ecosystem service capacity. And the distance of the annexing is inversely proportional. The closer the distance is, the stronger the annex will be.

\section{Introduction}

The survival of human societies is determined by the carrying capacity of their ecosystems $[1,2]$. The idea that natural resources and environments are free causes ecological resource development to exceed ecosystems' basic carrying capacities. This, in turn, leads to the reduction or complete loss of ecosystem service functions $[3,4]$. Now, more than two-thirds of services provided by ecosystems worldwide have already begun to decrease [5]; this trend may not be effectively reversed in the next 50 years $[3,4,6]$. Resource, ecological, and environmental crises have already seriously threatened China's development; for China to develop in a sustainable manner, scientific studies must establish macroscopic ecological security patterns and define key ecological spaces for prior protection. Therefore, exploring a scientific and effective method for delineating the range of ecosystem services is of positive significance in promoting the balanced development of ecosystem services and establishing and improving the ecosystem protection policy.

Ecosystem service functions are defined as "the natural benefits which human survival relies on and which are created and maintained by ecosystems and ecological processes" [7]. To describe ecosystem service functions more intuitively, Costanza et al. [8] conducted an assessment of ecosystem service values, which has become a key ecological research topic. Estoque and Murayama [9] found that the area of urban in the city of Baguio, the summer capital of the Philippines, expanded continuously during the study period, while the ecosystem service value dropped to $40 \%$ of that at the beginning of the study. Gómez-Baggethun and Barton [10] discovered that traditional monotonous urban planning is one-sided, and that a single socioeconomic indicator cannot assure the balanced ecological and economic development of 
a society. Moreover, introducing ecosystem service values has a positive impact on the development of human societies. Su et al. [11] primarily studied the Hangjiahu Plain agroecological zone, the Tianmu Mountain forest ecological zone, the Hangzhou urban ecological zone, and the Qiandao Lake Basin forest and wetland ecological zone and analyzed the effects of urbanization on regional ecosystems. Using research from around the world [12-17], Costanza et al. [6] summarized ecosystem service value assessments and further improved their evaluation. However, key research on ecosystem service values remains: for example, how should coverage of ecosystem services be delimited? How should a method of ecosystem service coverage quantification be developed?

Voronoi diagrams are partitioning that have been shown to be highly similar to some natural structures; as such, they have been studied extensively in fields including meteorology, geology, surveying and mapping, archaeology, molecular chemistry, ecology, and computer science [18, 19]. Voronoi diagrams are mainly applied to site selection and the determination of the scope of influence of different objects. Qin and Shi [20] utilized Voronoi diagrams to examine the spatial distribution of large-scale residential areas, ultimately making suggestions for planning in the region. Fan et al. [21] integrated Voronoi diagrams with spatial interaction models to investigate the Horqin Left Middle Banner, in Inner Mongolia, and proposed a method for improving this model by substituting town centrality for population size and integrating the model with weighted Voronoi diagrams. Traditional Voronoi diagrams are often used to describe the evenness of ground objects and are based on the principle of even distribution; however, they can better solve practical problems after some reasonable improvement. For example, introducing weights can compensate for the defects of traditional uniform Voronoi diagrams [22-25]. Li et al. [26] and Miao et al. [27] used Voronoi diagrams to examine parks in Shenyang and the wetland functional zone in Beijing, respectively, confirming the possibility of employing this method in ecosystem research. Nevertheless, there is still room for development in studying ecosystem services and enhancing its assessment accuracy.

To explore a suitable method of studying ecosystem service coverage areas, we introduce Voronoi diagrams and weighted Voronoi diagrams into ecosystem service value research and analyze ecosystem services coverage based on actual conditions. This approach helps quantify ecosystem service research not only at fixed points but also across regions. The Zhongyuan Urban Agglomeration is one of seven major urban agglomerations in China; it is the key development area of the Zhongyuan Economic Zone. Recent rapid social and economic development in this area has changed the types of ecosystems in the region, which in turn has led to changes in ecosystem service values in the coverage areas. In order to explore a reasonable method for estimating ecosystem service coverage areas, we will value the ecosystem services, that is, use economic methods to describe the strength of ecosystem services. Furtherly, we introduce the Voronoi diagram and the weighted Voronoi diagram to simulate and analyze the ecosystem service areas. The Voronoi diagram, the Euclidean distance map, and the rank assignment map are compared at various levels to explore the practicality of the weighted Voronoi diagram, thereby improving the spatially estimation practices of ecosystem service and providing the scope for future ecosystem service research.

\section{Study Area and Data Resources}

2.1. Study Area. The Zhongyuan Urban Agglomeration (shown in Figure 1) is located in central Henan Province, China; it has a total area of 58,700 square kilometers and includes nine cities: Zhengzhou, Luoyang, Kaifeng, Xinxiang, Jiaozuo, Xuchang, Luohe, Pingdingshan, and Jiyuan. This area is an important growth pole, not only of Henan Province but also of the Zhongyuan Economic Zone. In 2015, the gross national product of the agglomeration was $2.19 \times 10^{9}$ yuan and its population was $4.15 \times 10^{8}$. It has a nice natural environment with a wide distribution of varied land resources, a temperate monsoon climate, high topography in the west and north, and low topography in the east and south. The eastern part of the Zhongyuan Urban Agglomeration is located in the North China Plain, which is an essential grain production base and consists mainly of farmland. The Taihang Mountains in the west and north are important production sites of forest resources. Given the premise that ecological and economic development must be constantly promoted, it is necessary to protect regional ecologies to maintain balance in regional ecosystem services. Thus, we investigate the ecosystem service coverage of different ecosystems in the region and make suggestions for regional planning.

2.2. Data Sources. We aim to verify the applicability of weighted Voronoi diagrams to ecosystem service value research and place little emphasis on spatial and temporal variations in ecosystem service values. Weighted Voronoi diagrams of the ecosystem service value of the Zhongyuan Urban Agglomeration in 2015 are created to examine their meaning in ecosystem service research. Land use types significantly control the functions of regional ecosystem services and are commonly used as indictors of the values of regional ecosystem services [28]. We acquired land use map of the study area by interpreting Landsat OLI images in April 2015 , which is in the growing seasons and the images with low cloud cover percentages were selected. We used a manual interpretation method to derive land use maps from the remote sensing images. The land use is classified into four main types: farmland, forest, water, and construction area. To evaluate the accuracy of the land use map, we selected 110 random points from the map. The Land Use Atlas in Henan Province $(1: 1: 100000)$ and high-resolution satellite images from Google Earth were referred to validate the map. As a result, the overall accuracy of the land use map is $91.3 \%$, and the Kappa coefficient is 0.88 .

\section{Research Methods}

Based on Costanza's and Daly's [13] measurement and calculation of global ecosystem service values and the 


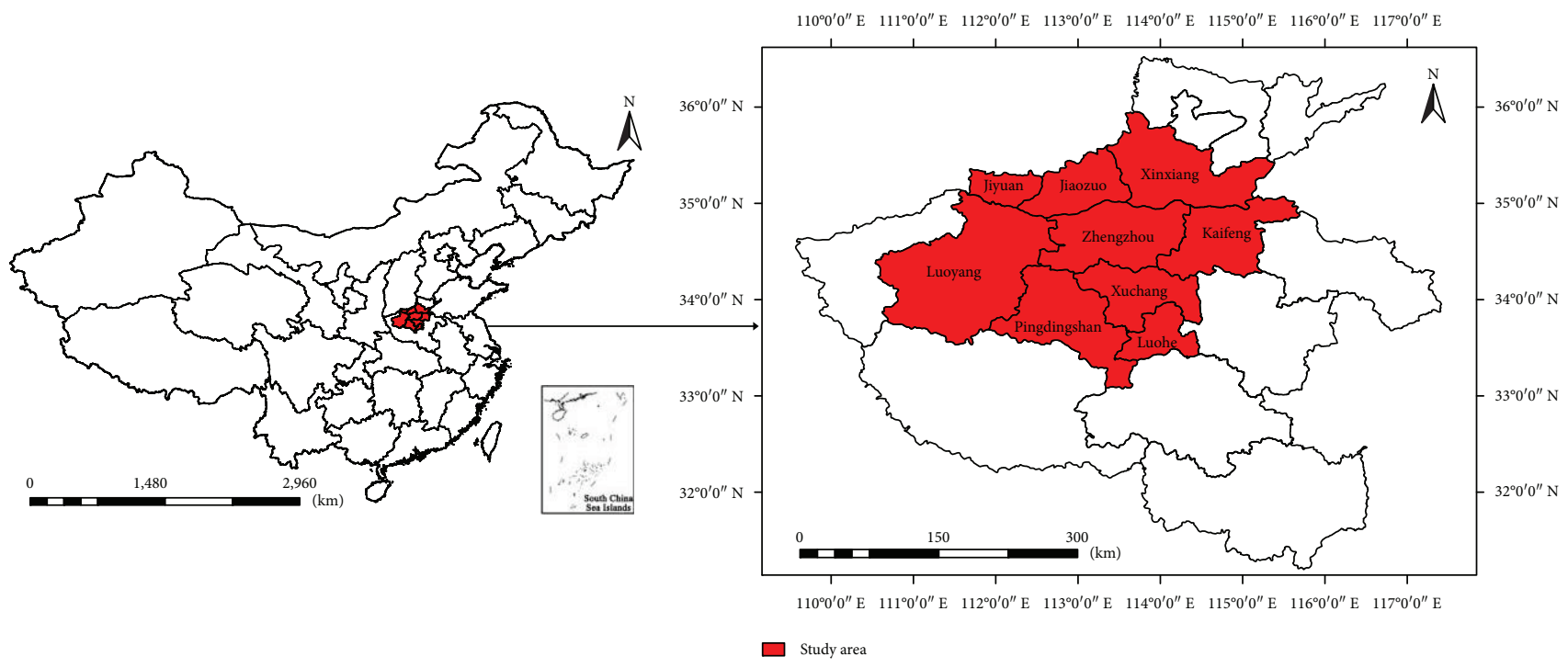

Figure 1: The location of the study area.

"Ecological Service Value Equivalence per Ecosystem Unit Area in China" table established by Xie at al. [29] based on actual conditions in China, this paper considers the actual condition of the Zhongyuan Urban Agglomeration to propose a novel research method, which is introduced into the correction coefficient of the study area's ecosystem service value. ArcGIS 10.1 and MATLAB are utilized to describe the spatial distribution of ecosystem service values in the Zhongyuan Urban Agglomeration. The results are compared in color gradings, traditional Voronoi diagrams, and weighted Voronoi diagrams to analyze these methods' advantages and drawbacks in ecosystem service value assessment (Figure 2) and provide a new perspective on ecosystem service value research.

3.1. The Correction Coefficient Model of Ecosystem Service Values. Based on previous research $[13,29,30]$ and realworld regional conditions, the assessment coefficient of ecosystem service values (as shown in Table 1) is improved. We propose an improved model based on grain yields (1) and a correction coefficient model of ecosystem service values (2).

$$
\begin{aligned}
E_{\mathrm{a}} & =\frac{1}{7}\left(\frac{m_{\mathrm{c}} p_{\mathrm{c}} q_{\mathrm{c}}}{M_{\mathrm{c}}}\right), \\
\mathrm{VC}_{i j} & =e_{i j} E_{\mathrm{a}},
\end{aligned}
$$

where $E_{\mathrm{a}}$ is the value of food supply services per unit agroecosystem per unit ecosystem (yuan), $m_{c}$ is the crop area $\left(\mathrm{hm}^{2}\right), q_{\mathrm{c}}$ is the crop yield per unit area $\left(\mathrm{kg} / \mathrm{hm}^{2}\right)$, $p_{c}$ is the notional average price of crops (yuan $/ \mathrm{kg}$ ), $M_{\mathrm{c}}$ is the total sown area $\left(\mathrm{hm}^{2}\right), \mathrm{VC}_{i j}$ denotes the equivalent value of ecosystem $i$ for function $j$, and $e_{i j}$ is the function of ecosystem service $j$ in the $i$ th ecosystem type relative to the food supply services provided by the agroecosystem. The $E_{\mathrm{a}}$ of the study area was calculated to be 1493.77

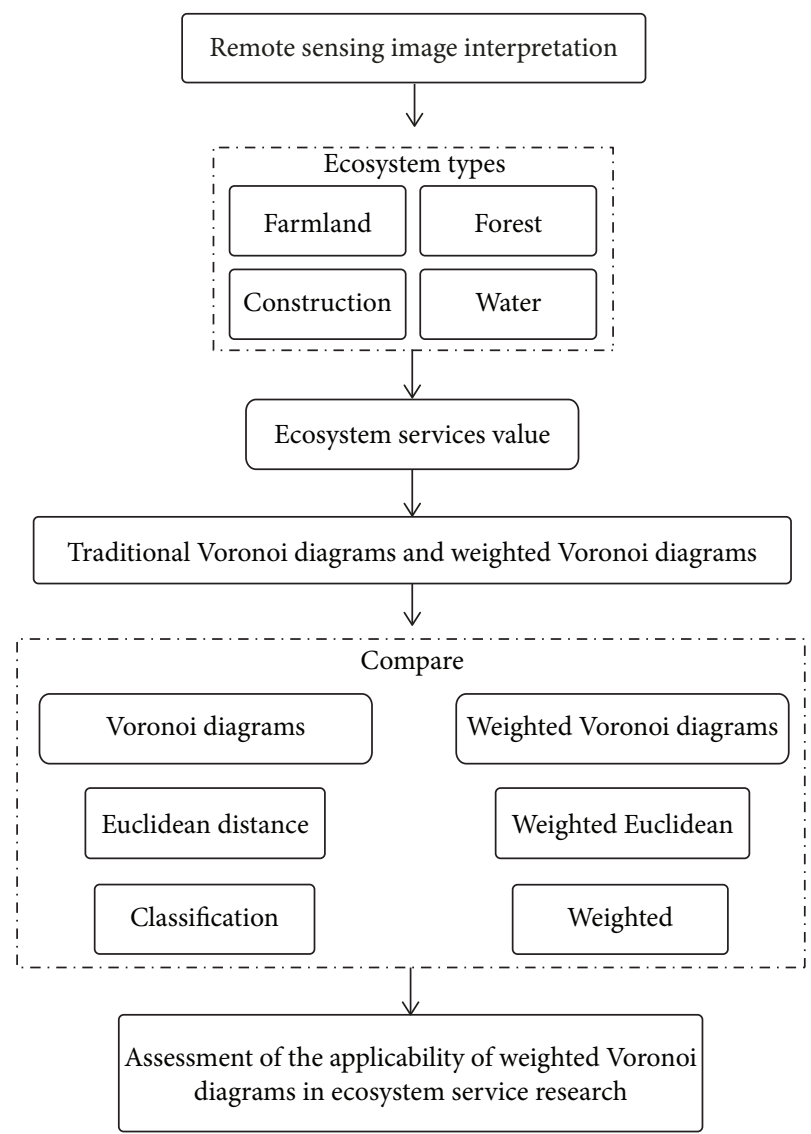

FIGURE 2: Research flowchart.

yuan $/ \mathrm{hm}^{2}$. The correction coefficient of ecosystem service value is listed in Table 1.

3.2. The Ecosystem Service Value (ESV) Model. The ecosystem service value (ESV) model is effective for estimating ecosystem service values based on different ecological types [30] and is expressed as illustrated in (3) and (4). 
TABLE 1: Correction coefficient of ecosystem services values $\left(\mathrm{y} / \mathrm{hm}^{2} \cdot \mathrm{a}\right)$.

\begin{tabular}{|c|c|c|c|c|}
\hline Service type & Forest ecosystem & Farm ecosystem & Water ecosystem & Construction ecosystem \\
\hline Gas regulation & 5228.19 & 746.88 & 0.00 & 0.00 \\
\hline Climate regulation & 4033.17 & 1329.45 & 687.13 & 0.00 \\
\hline Water conservation & 4780.06 & 896.26 & 30443.03 & -14325.25 \\
\hline Soil formation and protection & 5825.70 & 2180.90 & 14.93 & 0.00 \\
\hline Waste disposal & 1956.83 & 2449.78 & 27156.73 & -4660.56 \\
\hline Biodiversity conservation & 4869.69 & 1060.57 & 3734.42 & 0.00 \\
\hline Food production & 149.37 & 1493.77 & 448.13 & 0.00 \\
\hline Raw material & 3883.80 & 149.377 & 104.56 & 0.00 \\
\hline Entertainment culture & 1912.02 & 14.9377 & 8290.42 & 0.00 \\
\hline Total & 32638.87 & 10321.95 & 93674.31 & -18985.81 \\
\hline
\end{tabular}

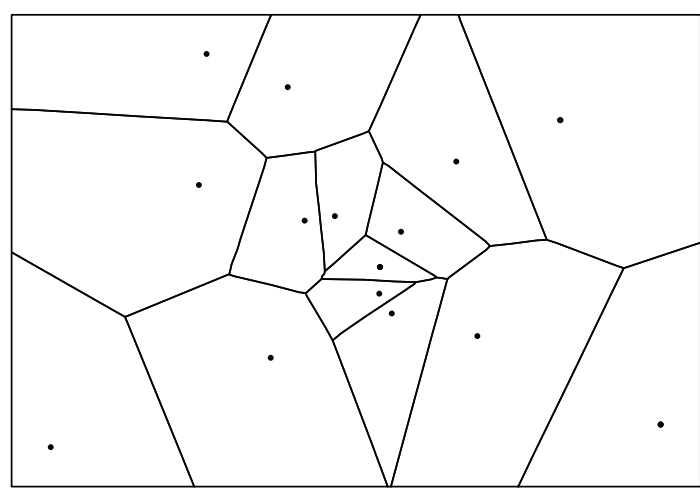

Figure 3: Traditional Voronoi diagram.

$$
\begin{aligned}
& \mathrm{ESV}_{i j}=A_{i} \times \mathrm{VC}_{i j}, \\
& \mathrm{ESV}_{i}=\sum_{j=1}^{n} \mathrm{ESV}_{i j},
\end{aligned}
$$

where ESV is the ecosystem service value (yuan), $A_{i}$ is the area of the $i$ th ecosystem type, and $\mathrm{VC}_{i j}$ denotes the equivalent coefficient of function $j$ for ecological land $i$.

3.3. Voronoi Diagrams. Voronoi diagrams (abbreviated as V diagrams, as shown in Figure 3) were proposed by Ukrainian mathematician Voronoi in 1908 and were applied to highdimensional spaces. By assuming that for a set of discrete points on Euclidean plane $P=\left\{p_{1}, p_{2}, \ldots, p_{n}\right\}$, where any two arbitrary points are noncoincident (i.e., $p_{i} \neq P_{j}, i \neq j$, $1 \leq i, j \leq n)$ and any four arbitrary points are nonconcyclic, $V\left(P_{1}\right) \cdots V\left(P_{i}\right)$ is calculated as

$$
V\left(P_{i}\right)=\bigcap_{i \neq j}\left\{P \mid d\left(P, P_{i}\right)<d\left(P, P_{j}\right)\right\} \quad j=1,2, \ldots, n,
$$

where $d\left(P, P_{i}\right)$ is the Euclidean distance between points $P$ and $P_{i}$. Thus, the distance from each point $P$ that belongs to $V\left(P_{i}\right)$ is closer to $P_{i}$ than any other points $P_{j}(i \neq j)$ of discrete point set $P$.

3.4. Weighted Voronoi Diagrams. Although Voronoi diagrams can depict the basic characteristics of some natural

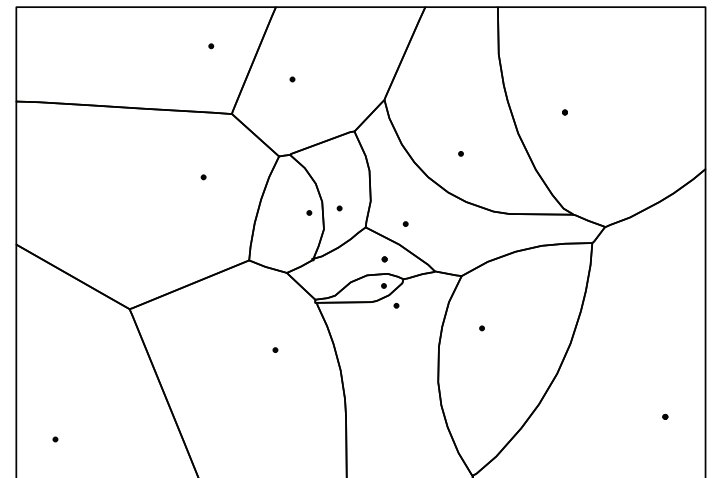

FIgURe 4: Weighted Voronoi diagram.

environments, they are unable to present the condition of some relatively complex ground objects. Based on traditional Voronoi diagrams, weighted Voronoi diagrams (as shown in Figure 4) introduce weight indices and convert traditional Euclidean distances into weighted Euclidean distances [31]. Given that $P=\left\{P_{1}, P_{2}, \ldots, P_{i}\right\}$, nonnegative real number weights $\lambda_{i}(i=1,2, \ldots, n)$ are introduced; hence, the weighted distance between points $P$ and $P_{i}$ is

$$
D\left(P, P_{i}\right)=\frac{d\left(P, P_{i}\right)}{\lambda_{i}} .
$$

Then, $V\left(P_{i}\right)$ should be revised to

$$
V\left(P_{i}\right)=\bigcap_{i \neq j}\left\{P \mid D\left(P, P_{i}\right)<D\left(P, P_{j}\right)\right\} \quad j=1,2, \ldots, n .
$$

When $\lambda_{1}=\lambda_{2}=\cdots=\lambda_{n}$, weighted Voronoi diagrams become traditional Voronoi diagrams.

\section{Results and Analysis}

4.1. Ecosystem Service Value Assessment. The Zhongyuan Economic Zone has lately been an important economic development region in China; more importantly, it is also the key region for stimulating the development of the central region of China and promoting balance in China's economic development. Because the Zhongyuan Urban Agglomeration is the key region of the Zhongyuan Economic Zone, 
TABLE 2: Comparison of different types of ecosystem service value coefficient.

\begin{tabular}{lcccc}
\hline Ecosystem service value coefficient & Forest ecosystem & Farm ecosystem & Water ecosystem & Construction ecosystem \\
\hline Costanza [13] $\left(\right.$ yuan $\cdot \mathrm{hm}^{-2} \cdot \mathrm{a}^{-1}$ ) & 8035.3 & 761 & 26487.4 & None \\
Xie et al. [29] $\left(\right.$ yuan $\cdot \mathrm{hm}^{-2} \cdot \mathrm{a}^{-1}$ ) & 12628.69 & 3547.89 & 44963.9 & None \\
Modified (yuan $\cdot \mathrm{hm}^{-2} \cdot \mathrm{a}^{-1}$ ) & 32638.87 & 10321.95 & 93674.31 & -18985.81 \\
\hline
\end{tabular}

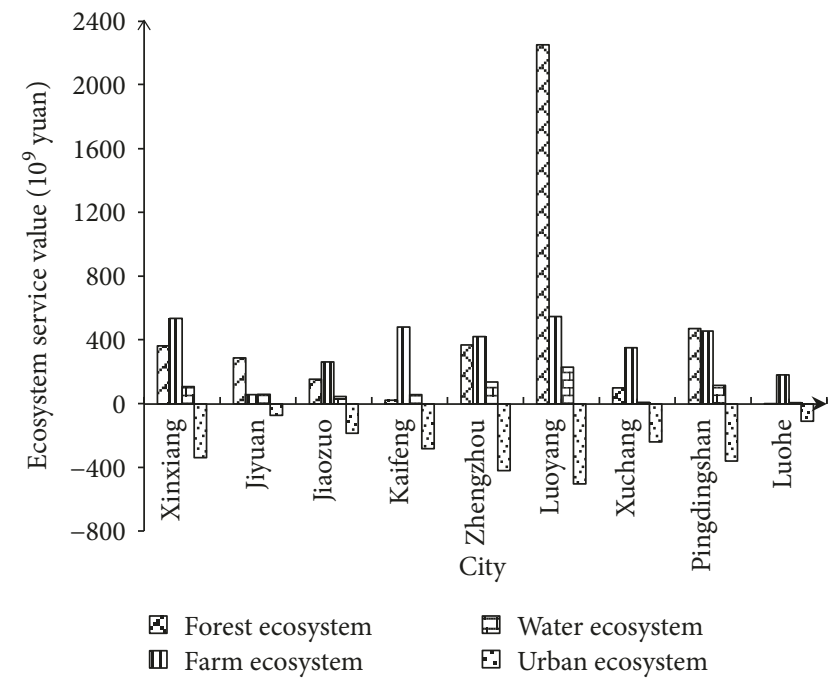

FIgURE 5: Ecosystem service values of nine cities in the Zhongyuan Urban Agglomeration.

its overall economic development acts as a model for the development of other regions. Therefore, proper ecosystem planning in the agglomeration and balanced ecological and economic development of the region enhances its competitiveness and encourages the development of other regions.

Before ecosystem service value assessment was completed, Costanza and Daly [13] and Xie et al. [29] performed coefficient correction of ecosystem service values according to real-world conditions in the study area. The results are listed in Table 2.

The coefficients of ecosystem service values in Table 2 and the ecosystem of different land use areas in the Zhongyuan Urban Agglomeration after satellite image interpretation were integrated to assess the ecosystem service value of each land use of the region. The results are shown in Figure 5.

The total ecosystem service value of the Zhongyuan Urban Agglomeration in 2015 was 575.7 billion yuan. The ecosystem service value of Luoyang was the highest, accounting for $43.93 \%$ of the regional total; in contrast, that of Luohe was only $1.42 \%$ of the regional total. Furthermore, the forest ecosystems of Luoyang contributed the most to the total ecosystem service value of the city, accounting for $89.09 \%$ of the total. The total ecosystem service value of Kaifeng was 28.259 billion yuan, of which the value of farm ecosystem was 48.216 billion yuan, whereas the loss in value caused by construction ecosystem expansion was up to 28.037 billion yuan. The ecosystem types combined with the administrative area could lead to the differences of ecosystem service values. Specifically, higher proportion of forest and water ecosystems in a region would increase the region's ecosystem service values and furtherly have positive influences upon the neighbor ecosystems.

4.2. Simulation of Ecosystem Service Coverage. Similar to social services, ecological services have coverage directly related to their intensity. Past ecosystem service studies could not determine the areas of influence of multiple service functions. For example, the ecosystem service function of climate regulation by forest ecosystems is affected by several factors and it is not possible to accurately assess specific service coverage. Hence, selecting an appropriate angle for measuring and calculating ecosystem service coverage can compensate for shortcomings in previous research.

Ecosystem service values quantify all kinds of ecosystem service functions and present them intuitively. The appearance of this variable allows ecosystem service coverage to be simulated and analyzed using an economic approach. The resulting Voronoi diagrams are shown in Figure 6 in a map showing a uniform distribution; however, there are considerable differences in ecosystem service values between cities. A uniform distribution cannot correctly depict the actual coverage of ecosystem services. Therefore, ecosystem service values of different regions are presented as weighted Voronoi diagrams, which partly compensate for defects in the expression of service coverage by traditional Voronoi diagrams.

The ecosystem service values of all cities in the Zhongyuan Urban Agglomeration were used as weight indices to improve the original Voronoi diagrams, as illustrated in Figure 7. These results, together with the data in Figure 5, demonstrate that the ecosystem service value of Luoyang accounts for a significant portion of the total ecosystem service value of the Zhongyuan Urban Agglomeration. Its influence is considerably greater than those of other cities. Integration of the different service boundaries of Figures 5 and 7 shows that, taking Luoyang as an example, the ecosystem service value coverage of the city expands significantly, which is highly related to the larger ecosystem service value of the city. For regions with lower service values, such as Jiyuan and Luohe, except for certain preserved coverage, areas are impacted by regions with greater ecosystem service values. That is, if the ecosystem service value is greater, the service coverage is more extensive. To an extent, these areas affect and compensate for areas with smaller ecosystem service values.

To highlight the interactions and relationships between ecosystem services of different regions, we classified ecosystem service coverage using the Jenks natural breaks classification method into five types: high-, medium to high-, medium-, medium to low-, and low-impact areas. The traditional Voronoi diagrams are compared with the weighted ones in Figure 8. 


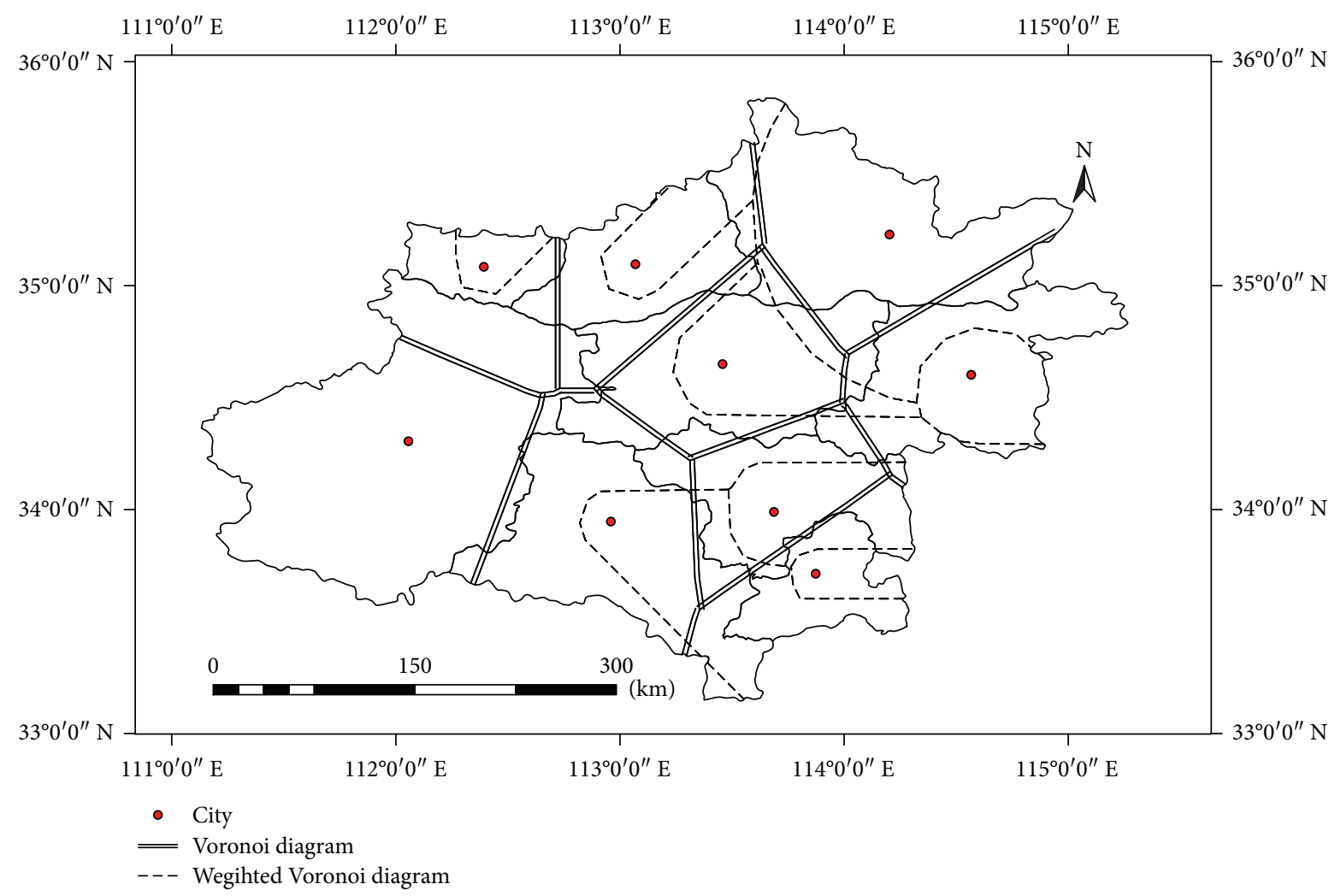

Figure 6: Comparison of the traditional and weighted Voronoi diagrams of the study area.

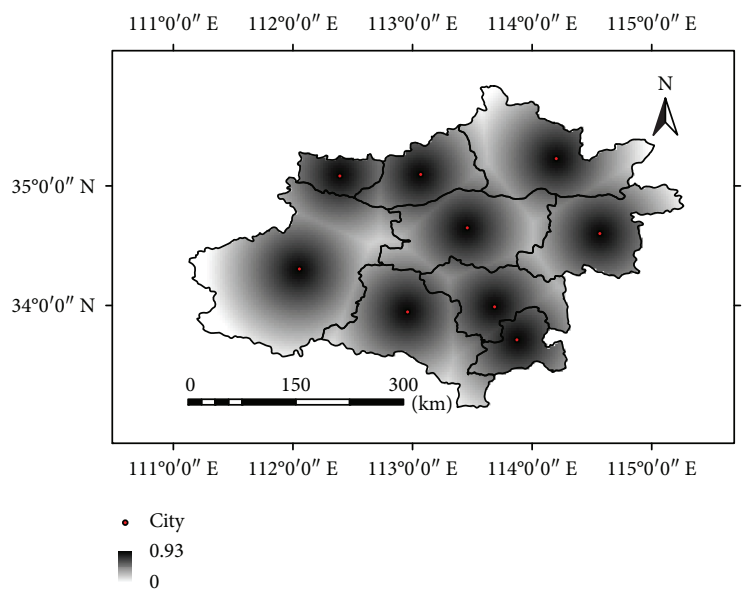

(a)

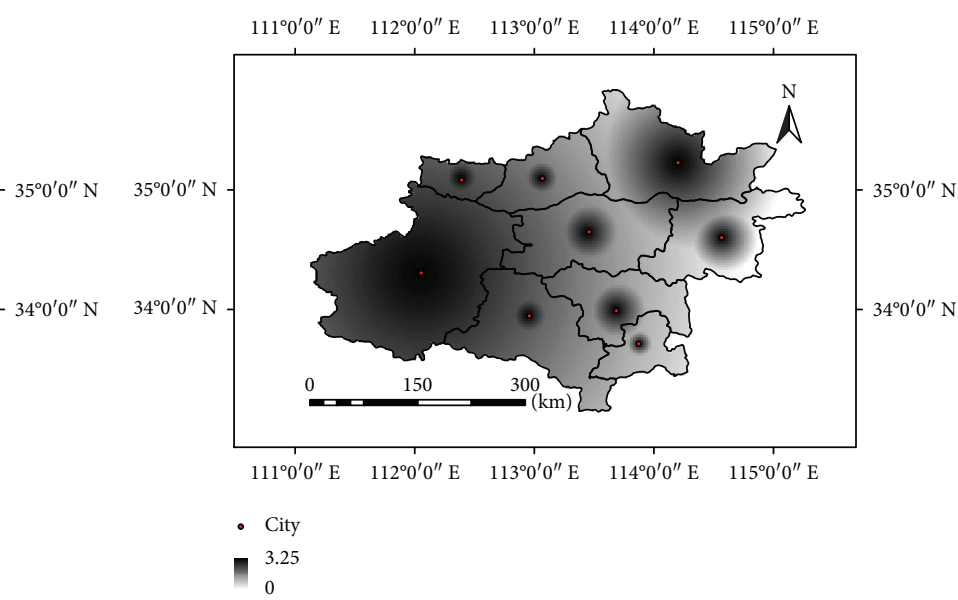

(b)

Figure 7: Comparison of Euclidean distances between (a) the traditional Voronoi diagrams and (b) weighted Voronoi diagrams of the study area.

As shown in Figure 8, regions may have various effects on each other, although the center of each region is mainly influenced by the region itself. In addition, regions with greater ecosystem service capabilities compensate for regions with lower capabilities, which conceal the originally insignificant ecosystem service capabilities of regions with lower service capacities. The ecosystem service interactions engulfing different regions can be monitored and controlled artificially. Rational distribution of ecosystems over the study area will considerably enhance a region's ecosystem service capability and thus its ecosystem service quality. If the stepwise progressive decreases of different kinds of ecosystem service capabilities can be successfully determined, it would be possible to more accurately evaluate the influences and coverage of ecosystem services.

From the total ecosystem service value in Figure 8, the type of ecosystem that dominates the total ecosystem services can be seen and various types of ecosystem service coverage 


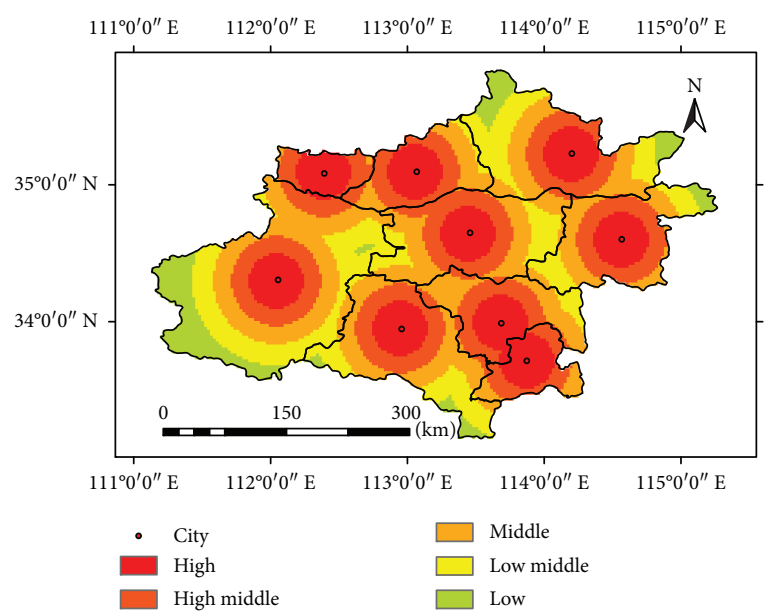

(a)

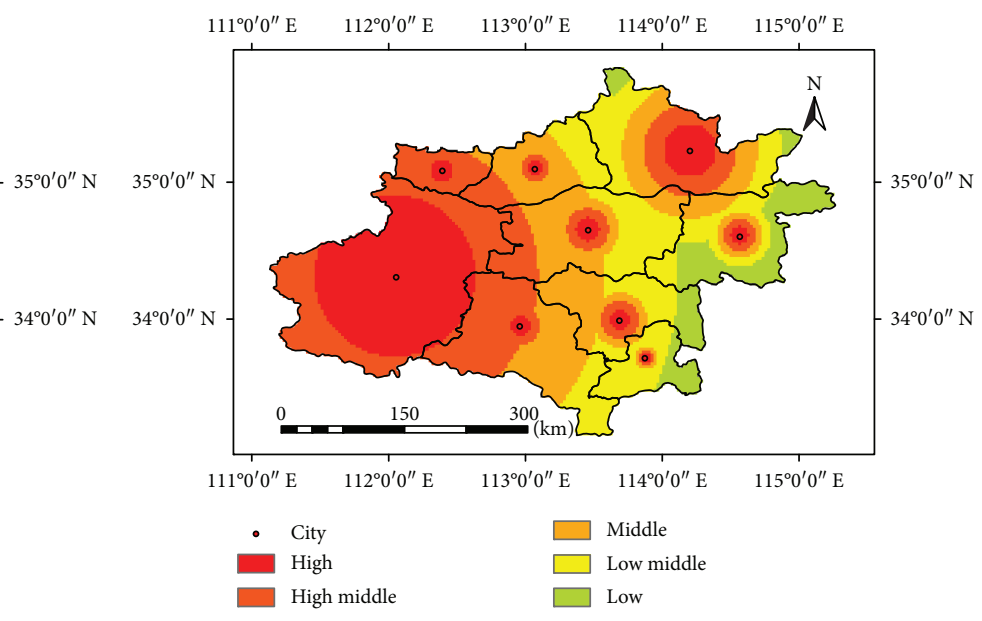

(b)

FIGURE 8: Comparison of ecosystem service classification between (a) traditional Voronoi diagrams and (b) weighted Voronoi diagrams of the study area.

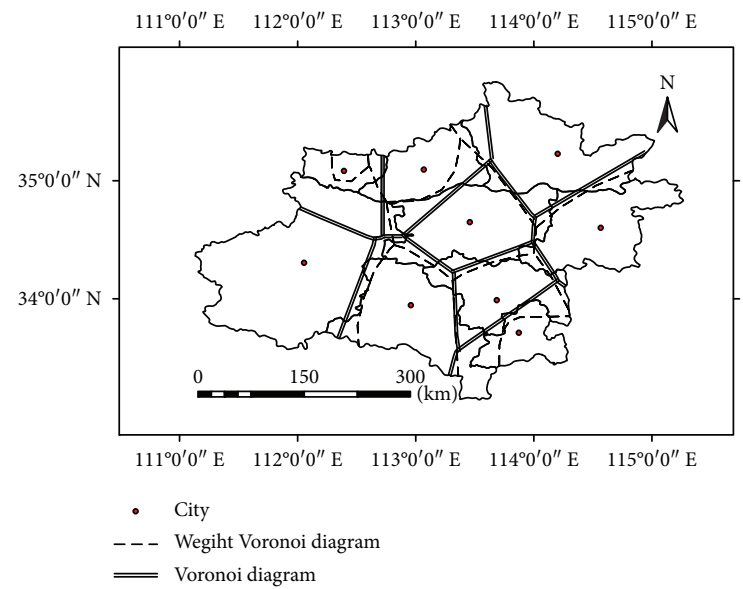

(a)

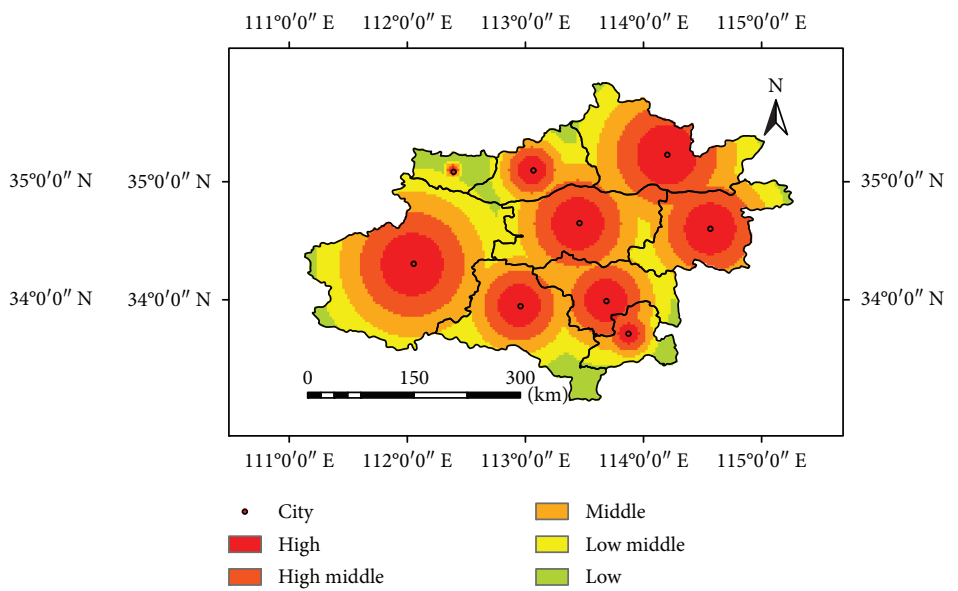

(b)

FIGURE 9: Service impact ranges of (a) traditional Voronoi diagram and (b) weighted Voronoi diagram in farmland ecosystem.

can be studied. Thus, the uneven distribution of land use types between regions can be obtained. We illustrate the ecosystem service capabilities and coverage of farm ecosystem, forest ecosystems, water ecosystems, and construction ecosystems of regions in the Zhongyuan Urban Agglomeration in Figures 9-12.

Excluding Jiyuan, Luohe, and Jiaozuo, the farmland ecosystem service values of the study area were 40-60 billion yuan. According to Figures 9-12, it is obvious that regions with less farmland ecosystem service capability are more considerably affected by regions with greater capabilities. For regional division, compared to traditional Voronoi diagrams, the weighted Voronoi diagrams also consider the actual spatial distribution of farmland. Additionally, compared to the traditional Voronoi diagrams, which use color grading to represent ecosystem service values, the weighted diagrams reflect the progressive reduction over space and the nonuniformity of ecosystem services, more closely representing reality. Traditional ecosystem service value evaluation only considers the ecosystem service capabilities of cities, but not the direct interactions between cities, whereas weighted Voronoi diagrams can better explain these interactions. Construction ecosystems cause negative ecosystem service values and lower overall ecosystem service capabilities. Therefore, during calculation, all construction was assigned positive values. High-impact areas are those that are more greatly affected by construction ecosystems. The ecosystem service value losses of these areas are significantly higher than those of other areas. Areas with higher levels of urbanization and more extensive construction experience stronger reduction. In the study area, there are more forest ecosystems in the west and fewer in the east. Thus, areas with higher forest ecosystem service capabilities impose greater influences on areas with lower capabilities. Luoyang has vast forest ecosystems. Strong forest ecosystem service capability is the basic characteristic of the city's ecosystem services; Luoyang's ecosystem service value is $6.91,7.32,15.06,119.42,10.15,27.3$, 27.3, and 323.29 times of those of Xinxiang, Jiyuan, Jiaozuo, 


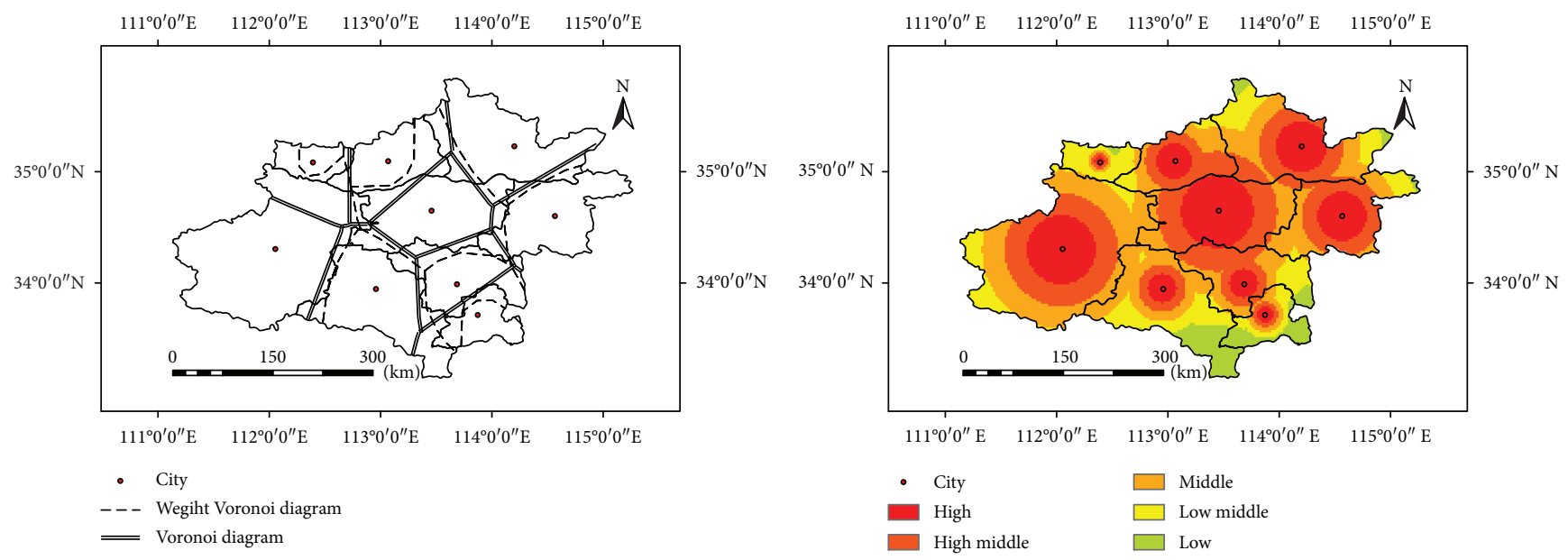

(a)

(b)

FIGURE 10: Service impact ranges of (a) traditional Voronoi diagram and (b) weighted Voronoi diagram in construction ecosystem.

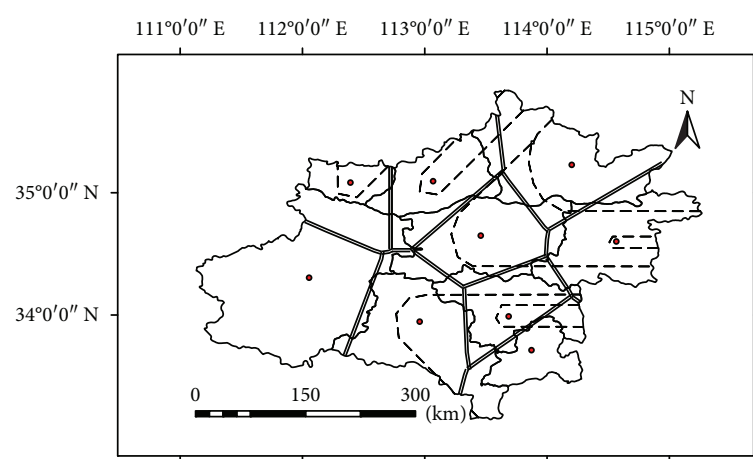

$111^{\circ} 0^{\prime} 0^{\prime \prime} \mathrm{E} \quad 112^{\circ} 0^{\prime} 0^{\prime \prime} \mathrm{E} \quad 113^{\circ} 0^{\prime} 0^{\prime \prime} \mathrm{E} \quad 114^{\circ} 0^{\prime} 0^{\prime \prime} \mathrm{E} \quad 115^{\circ} 0^{\prime} 0^{\prime \prime} \mathrm{E}$

- City

- - - Wegiht Voronoi diagram

Voronoi diagram

(a)

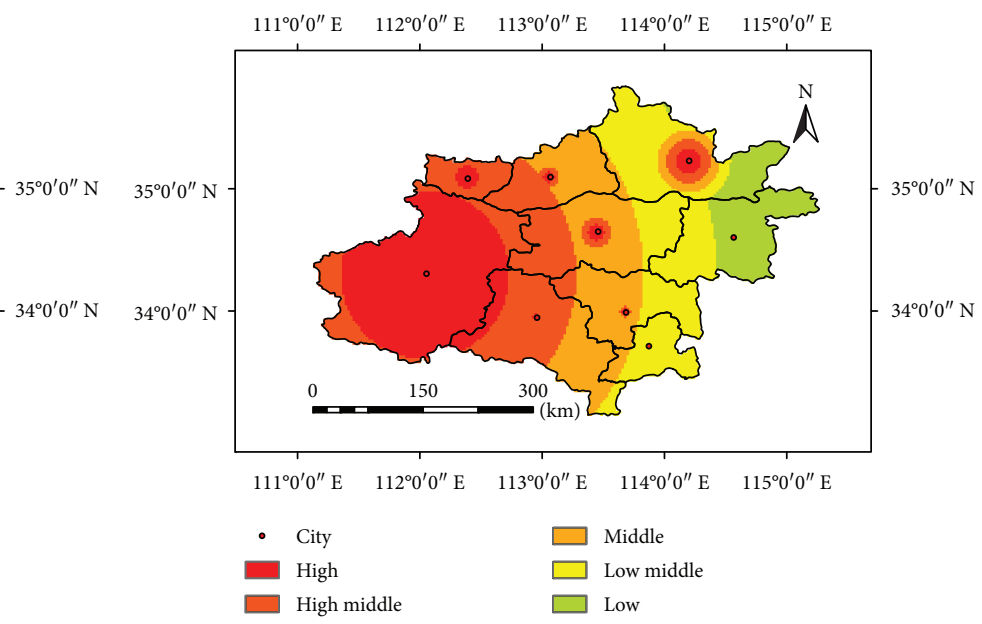

(b)

FIGURE 11: Service impact ranges of (a) traditional Voronoi diagram and (b) weighted Voronoi diagram in forest ecosystem.

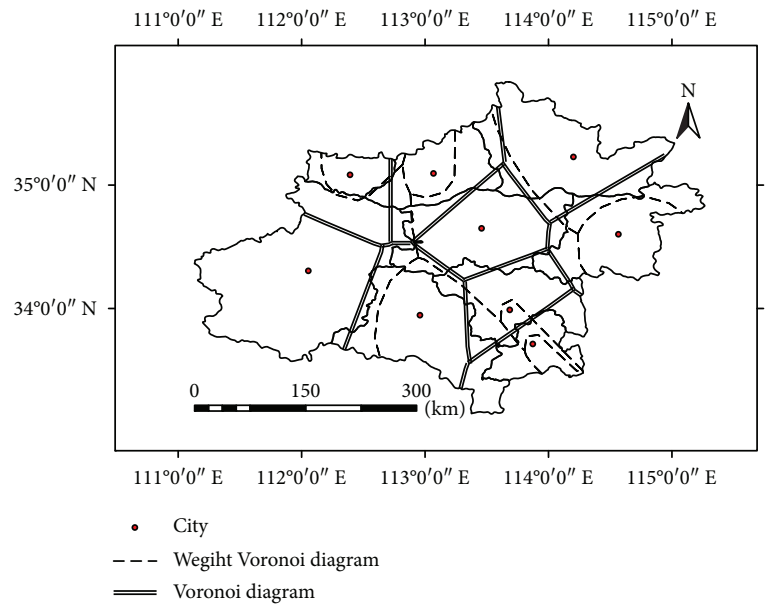

(a)

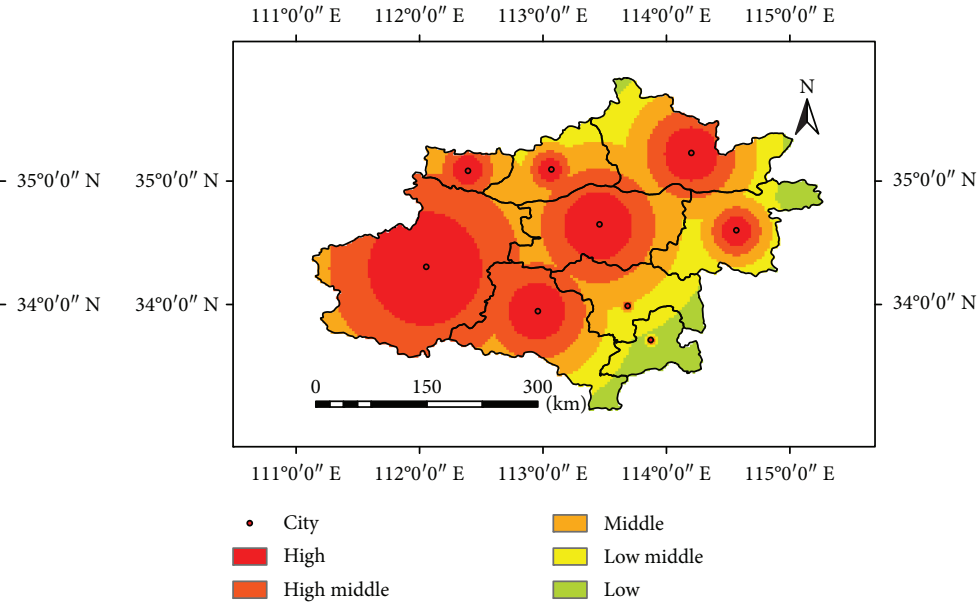

(b)

FIGURE 12: Service impact ranges of (a) traditional Voronoi diagram and (b) weighted Voronoi diagram in water ecosystem. 


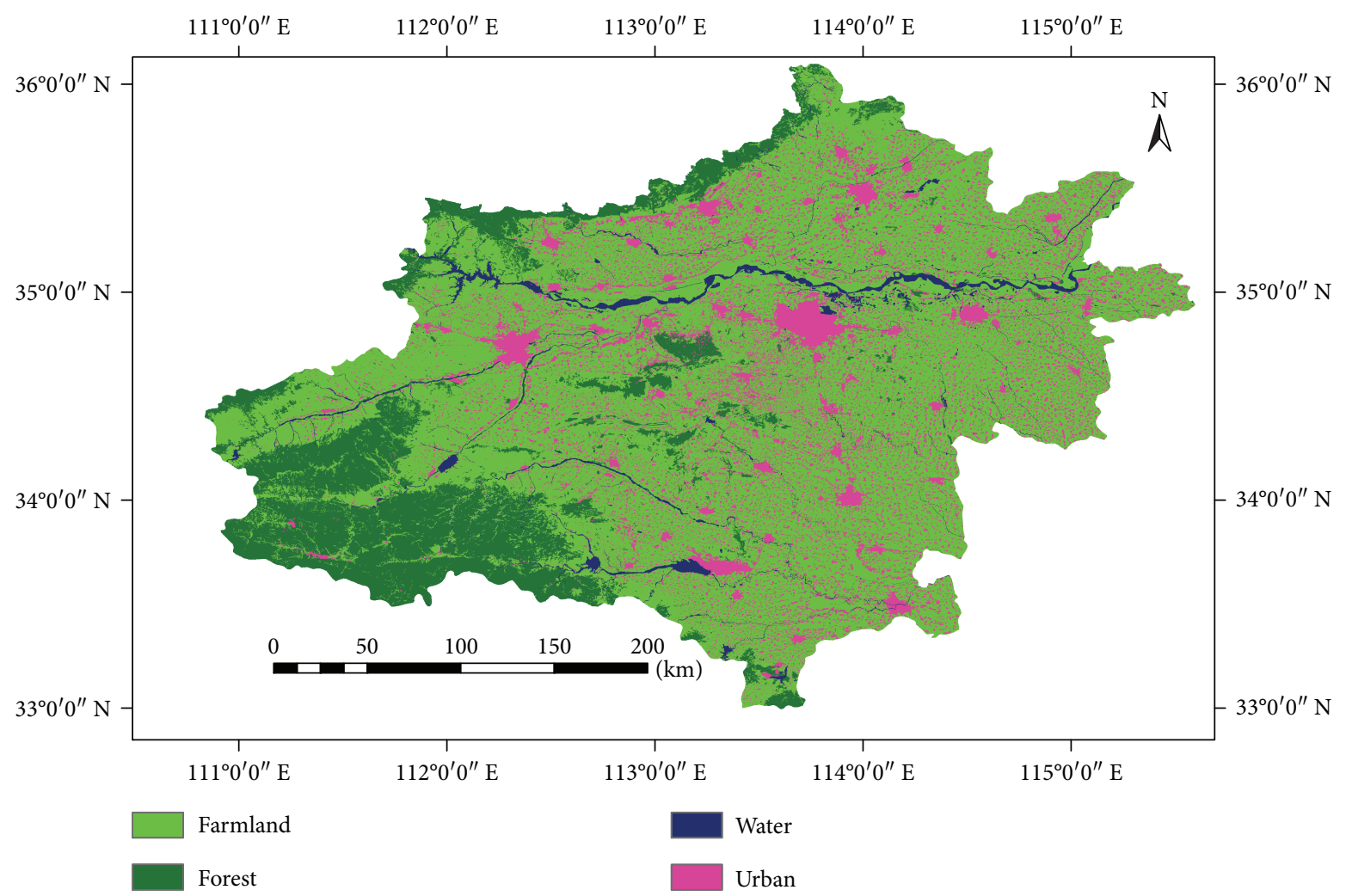

Figure 13: Remote sensing image interpretation data of the Zhongyuan Urban Agglomeration in 2015.

Kaifeng, Zhengzhou, Xuchang, Pingdingshan, and Luohe, respectively. Engulfing effects and compensation due to the forest ecosystem services of Luoyang gradually weaken with distance. However, the low-impact forest ecosystem service area of the city still has relatively significant effects on other areas. The weighted Voronoi diagrams, compared to the traditional Voronoi diagrams, have the advantage of intuitively presenting ecosystem service capabilities and their coverage over space. The coverage of water ecosystem services and the actual distribution of water ecosystems are relatively strongly correlated. The Yellow River runs through the central and the central-north regions of the Zhongyuan Urban Agglomeration. Rivers in Luoyang are widely distributed, which places Luohe in a low-impact water ecosystem service area. This distribution of high-impact areas in the northwest and low-impact areas in the northeast matches the satellite image interpretation results. Comparison between the satellite image interpretation (Figure 13) and ecosystem service coverage map reveals that high-, medium-, and low-impact ecosystem service areas are directly related to the distinct types of ecosystems in different cities, further confirming that weighted Voronoi diagrams are suitable for simulation of ecosystem service coverage and provide a new method and direction for these studies.

\section{Discussion and Conclusion}

5.1. Discussion. This study employs cities as central points for investigating ecosystem service coverage. This method considers an entire region and thereby presents the spatial distribution of ecosystem services, providing a new perspective for ecosystem service coverage research. Nevertheless, this method neglects nonuniform ecosystem distributions, ecosystem distribution within the region, and the interactions between ecosystems, which reduce the accuracy of ecosystem service coverage research. Thus, in future studies, better results can be obtained if fixed point studies can be conducted for different land use types.

Ecosystem service capabilities were classified using the Jenks natural breaks classification method. Although this method can partly reveal the basic features of research topics and data, it neglects the gradual reduction in service distance of service providers and the acceptance capabilities of service. Proper investigation of the gradual decrease of different ecosystem services over distance, and the acceptance rates of different service providers, allows the new method to be better applied to the actual production activities.

5.2. Conclusions. The total ecosystem service value of the Zhongyuan Urban Agglomeration in 2015 was 575.7 billion yuan. The total ecosystem service value of Luoyang was the highest, at 267.665 billion yuan; that of Luohe was the lowest, at only 13.881 billion yuan. The differences between regions are huge. Forest ecosystems have the greatest ecosystem service value per unit area - up to 32.639 thousand yuan per hm. Meanwhile, construction ecosystem service values significantly reduce the service values of different ecosystem types. Continuous expansion of construction ecosystems has 
decreased the ecosystem service value of Zhengzhou by 37.012 billion yuan, with the most remarkable decline seen in Kaifeng.

Compared to traditional Voronoi diagrams, weighted Voronoi diagrams are more suitable for studying ecosystem service coverage. Comparison between the two indicates that the spatial coverage of ecosystem services presented by the weighted Voronoi diagrams better fit actual conditions. Traditional Voronoi diagrams describe uniform ecosystem services and neglect the discrepancies in actual service coverage caused by regional differences. In weighted Voronoi diagrams, regions with higher ecosystem service capabilities partly engulf and compensate for regions with lower capabilities. These engulfing and compensating actions are inversely proportional to the distance between two regions. When two ecosystems are closer to each other, their interactions are stronger. The region with lower capability is more easily engulfed by the region with higher capability, and the ecosystem services of the former are enhanced. These actions partly contribute to the spatial and temporal distribution of ecosystems in the region and promote the union of different regions, thereby enhancing overall regional ecosystem service capability.

The ecosystem service coverage of farm ecosystem, forest ecosystems, water ecosystems, and construction ecosystems was studied separately, and the results compared to actual conditions. We found that the two agree relatively well, again proving that weighted Voronoi diagrams perform well in assessing ecosystem service coverage. The service coverage of different ecosystem types was also examined. We discovered that, for larger differences in ecosystem type and size between different regions, weighted Voronoi diagrams differ more significantly than their traditional counterparts. This result demonstrates that, for two regions whose ecosystem service differences are relatively large, traditional Voronoi diagrams do not match actual service coverage.

\section{Data Availability}

The readers can contact the authors to access the data in the manuscript.

\section{Disclosure}

The content does not represent the official position of the Chinese government and is entirely the responsibility of the authors.

\section{Conflicts of Interest}

The authors declare that they have no conflicts of interest.

\section{Acknowledgments}

This paper was supported by the National Natural Science Foundation of China (41601175 and 41771380). The Key Scientific Research Project of Henan Province (16A610001), supported by the Program for Innovative Research Team (in Science and Technology) in the University of Henan
Province (16IRTSTHN012), the GDAS’ Project of Science and Technology Development (2017GDASCX-0101 and 2018GDASCX-0904), the Guangdong Innovative and Entrepreneurial Research Team Program (2016ZT06D336), the Science and Technology Program of Guangdong Province (2017B010117008), and the Science and Technology Program of Guangzhou (201604016047).

\section{References}

[1] D. E. Campbell, "Emergy analysis of human carrying capacity and regional sustainability: an example using the state of Maine," Environmental Monitoring and Assessment, vol. 51, no. 1/2, pp. 531-569, 1998.

[2] E. S. Nakajima and E. Ortega, "Carrying capacity using emergy and a new calculation of the ecological footprint," Ecological Indicators, vol. 60, pp. 1200-1207, 2016.

[3] N. M. Haddad, L. A. Brudvig, J. Clobert et al., "Habitat fragmentation and its lasting impact on Earth's ecosystemse1500052," Science Advances, vol. 1, no. 2, 2015.

[4] N. M. Haddad, L. A. Brudvig, J. Clobert et al., "Habitat fragmentation and its lasting impact on Earth's ecosystems," Science Advances, vol. 1, no. 2, article e1500052, 2015.

[5] Millennium Ecosystem Assessment, Ecosystems and Human Well-Being; Biodiversity Synthesis, WorldResources Institute, Washington DC, 2005.

[6] R. Costanza, R. de Groot, P. Sutton et al., "Changes in the global value of ecosystem services," Global Environmental Change, vol. 26, no. 1, pp. 152-158, 2014.

[7] Z. Ouyang, T. Zhao, X. Wang, and H. Miao, "Ecosystem services analyses and valuation of China terrestrial surface water system," Acta Ecologica Sinica, vol. 24, no. 10, pp. 2091-2099, 2004.

[8] R. Costanza, R. d'Arge, R. de Groot et al., "The value of the world's ecosystem services and natural capital," Nature, vol. 387, no. 6630, pp. 253-260, 1997.

[9] R. C. Estoque and Y. Murayama, "Landscape pattern and ecosystem service value changes: implications for environmental sustainability planning for the rapidly urbanizing summer capital of the Philippines," Landscape and Urban Planning, vol. 116, pp. 60-72, 2013.

[10] E. Gómez-Baggethun and D. N. Barton, "Classifying and valuing ecosystem services for urban planning," Ecological Economics, vol. 86, pp. 235-245, 2013.

[11] S. Su, R. Xiao, Z. Jiang, and Y. Zhang, "Characterizing landscape pattern and ecosystem service value changes for urbanization impacts at an eco-regional scale," Applied Geography, vol. 34, pp. 295-305, 2012.

[12] J. F. Barlow, "A change effects all the organisms in the ecosystem," South Dakota Journal of Medicine, vol. 47, no. 6, p. 186, 1994.

[13] R. Costanza and H. E. Daly, "Natural capital and sustainable development," Conservation Biology, vol. 6, no. 1, pp. 37-46, 1992.

[14] E. Matthews, "Global vegetation and land use: new highresolution data bases for climate studies," Journal of Applied Meteorology, vol. 22, no. 3, pp. 474-487, 1983.

[15] D. Pauly and V. Christensen, "Primary production required to sustain global fisheries," Nature, vol. 374, no. 6519, pp. 255257, 1995. 
[16] H. Jack Ruitenbeek, "Functions of nature: evaluation of nature in environmental planning management and decision making," Ecological Economics, vol. 14, no. 3, pp. 211-213, 1995.

[17] J. H. Ryther, "Photosynthesis and fish production in the sea," Science, vol. 166, no. 3901, pp. 72-76, 1969.

[18] J. Liu, X. Sun, and S. Liu, "Weighted node network Voronoi diagram and its application to optimization of chain stores layout," International Journal of Machine Learning and Cybernetics, vol. 7, no. 4, pp. 679-688, 2016.

[19] A. Okabe, B. Boots, and K. Sugihara, "Nearest neighbourhood operations with generalized Voronoi diagrams: a review," International Journal of Geographical Information Science, vol. 8, no. 1, pp. 43-71, 1994.

[20] Y. Qin and X. Y. Shi, "Optimizing spatial distribution of residential areas based on Voronoi diagram," Science of Surveying and Mapping, vol. 1, pp. 136-138, 2012.

[21] Q. Fan, H. X. Zhang, Y. H. Li, and J. X. Wang, "Quantitative analysis and urban system planning of county area based on spatial-interaction model: a case in Horqin Left Middle Banner," Inner Mongolia, Scientia Geographica Sinica, vol. 34, no. 5, pp. 601-607, 2014.

[22] C. Xy, L. Jp, L. Chen, and H. Yj, "Intraspecific and interspecific competition analysis of Cunninghamia lanceolata ecological forest based on weighted Voronoi diagram," Chinese Journal of Ecology, vol. 35, no. 9, pp. 2553-2561, 2016.

[23] J. Y. Gu, Y. G. Zhou, and L. M. Guo, "Urban influence regions and spatial relation of Beijing-Tianjin Hebei region based on weighted Voronoi diagram and breakpoint theory," Geography and Geo-Information Science, vol. 30, no. 1, pp. 65-69, 2014.

[24] X. W. Qin, S. Q. Zhang, X. F. Li, X. D. Na, X. Pan, and H. Yu, "Assessment of red-crowned crane's habitat suitability based on weights-of-evidence," Acta Ecologica Sinica, vol. 29, no. 3, pp. 1074-1082, 2009.

[25] S. Xie, X. Feng, J. Wang, and W. Lu, "Radiation domain of commercial centers in Nanjing based on analysis of road network weighted Voronoi diagram," Acta Geographica Sinica, vol. 64, no. 12, pp. 1467-1476, 2009.

[26] X. M. Li, C. F. Liu, and W. Wu, "Recreational pressure of urban parks in Shenyang," Chinese Journal of Ecology, vol. 28, no. 5, pp. 992-998, 2009.

[27] L. L. Miao, W. G. Jiang, S. D. Wang, and L. Zhu, "Comprehensive assessments and zoning of ecological service functions for Beijing wetland based on RS and GIS," Remote Sensing for Land and Resources, vol. 25, no. 3, pp. 102-108, 2013.

[28] P. H. Verburg, K. H. Erb, O. Mertz, and G. Espindola, "Land system science: between global challenges and local realities," Current Opinion in Environmental Sustainability, vol. 5, no. 5, pp. 433-437, 2013.

[29] G. D. Xie, L. Zhen, C. X. Lu, Y. Xiao, and C. Chen, "Expert knowledge based valuation method of ecosystem services in China," Journal of Natural Resources, vol. 23, no. 5, pp. 911919, 2008.

[30] F. Zhou, S. Y. Chen, and M. F. Wu, "Land utility structure changing trend and its response to ecological service functions-a case study in Zhanjiang City," Journal of Safety and Environment, vol. 7, no. 5, pp. 76-79, 2007.

[31] S. M. Feng, "Study on service coverage of distribution center based on Voronoi diagram," in 2009 International Conference on Information Engineering and Computer Science, vol. 29no. 3, pp. 142-144, Wuhan, China, December 2009. 


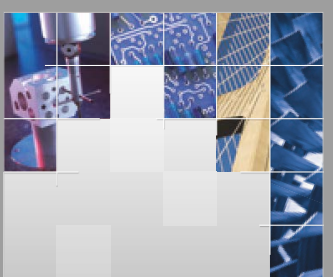

\section{Enfincering}
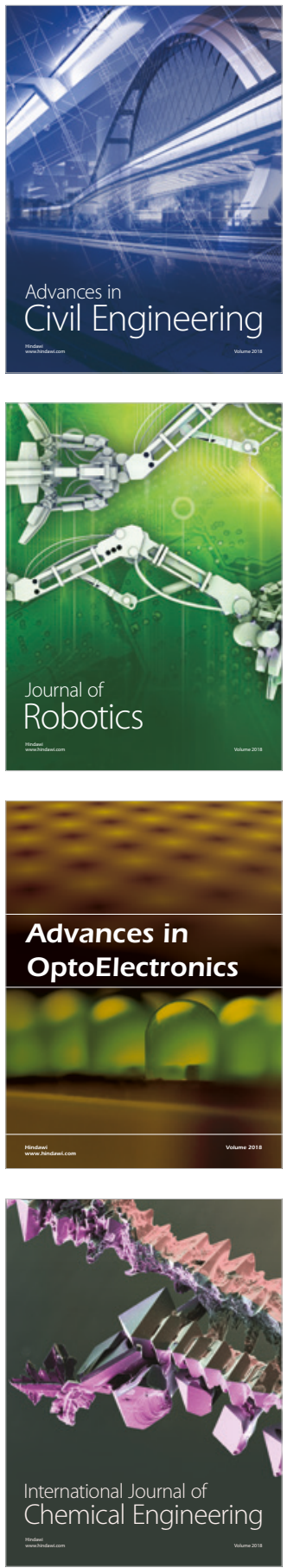

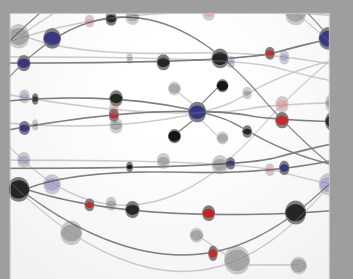

\section{Rotating \\ Machinery}

The Scientific World Journal

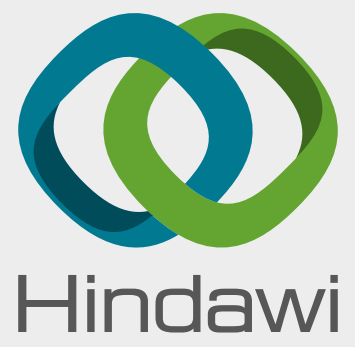

Submit your manuscripts at

www.hindawi.com
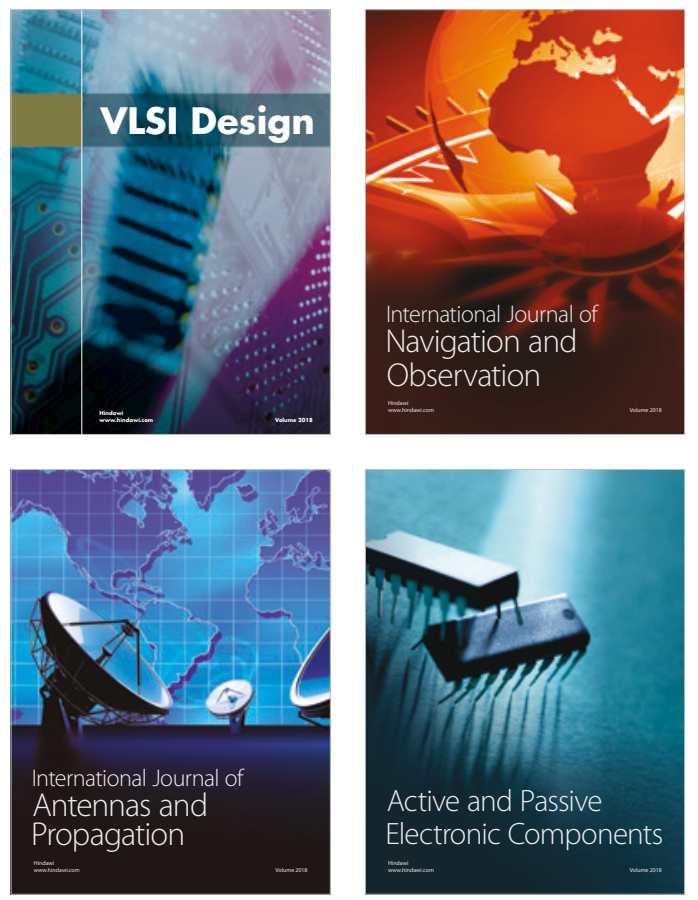
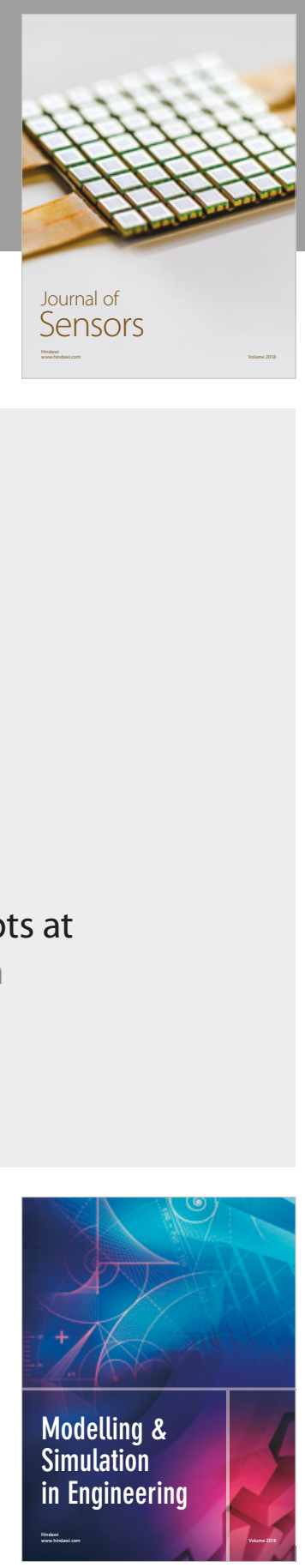

\section{Advances \\ Multimedia}
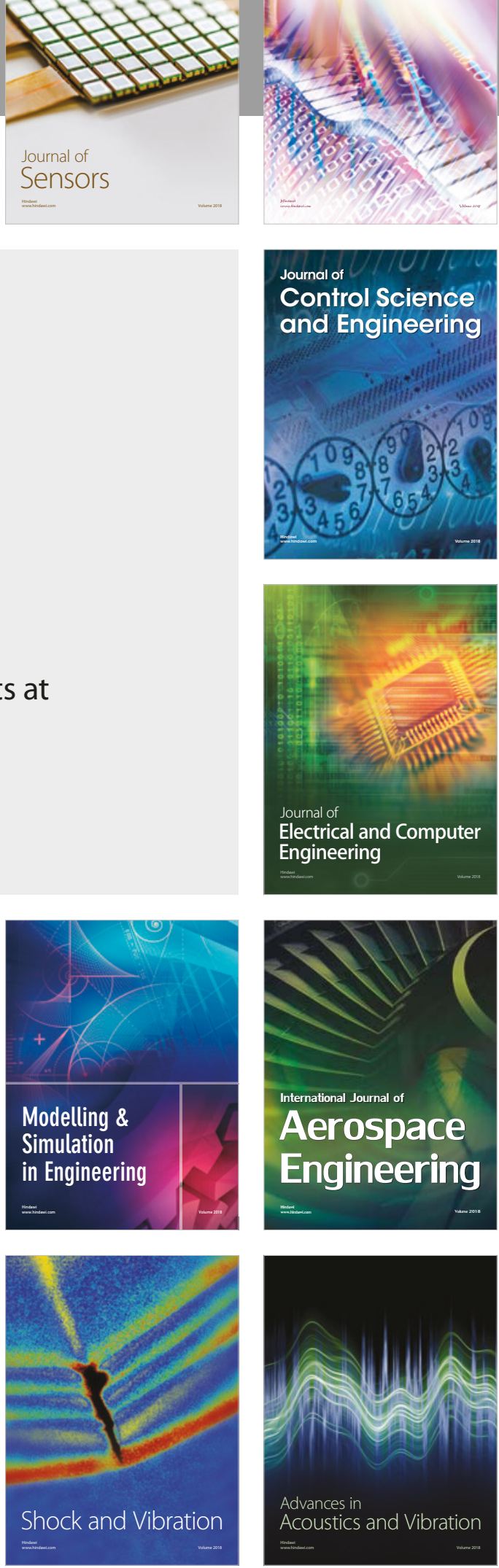